Journal of Engineering and Applied Sciences 14 (Special Issue 8): 10349-10359, 2019

ISSN: $1816-949 \mathrm{X}$

(C) Medwell Journals, 2019

\title{
Nonlinear Vibration and Dynamic Analysis of Functionally Graded Graphene-Reinforced Eccentrically Stiffened Imperfection Composite Cylindrical Shell Panels
}

\author{
Hamad M. Hasan and Satar A. Mutlak \\ Department of Mechanical Engineering, College of Engineering, \\ University of Anbar, Ramadi, Iraq
}

\begin{abstract}
This study researches the nonlinear vibration and dynamic response of Functionally Graded Graphene Platelets (FG-GPLs) reinforce eccentrically stiffened imperfection cylindrical Shellpanels. The governing equations are derived in the frame work of the classical Shelltheory coupled with the Von Karman nonlinearity terms. The effective modulus of elasticity for the composite panels and stiffeners is determined with the Halpin-Tsai technique while the rule of mixture is utilized to evaluate the Poisson's ratio and mass density. Galerkin approach coupled with the Runge-Kutta method is employed to solve the nonlinear equation for the dynamic analysis of the Shellpanels. A comparative study is carried out with that available in the open literature to prove the validation of the obtained results. In numerical results, the influence of reinforcing the stiffeners with GPLs, weight fraction, GPLs distribution pattern, geometric parameters of stiffeners, imperfection factor and exciting force on the nonlinear dynamic response of the Shellpanels is analysed in details.
\end{abstract}

Key words: Nonlinear vibration, dynamic analysis, functionally graded graphene platelets, Shellpanels, eccentrically stiffeners, dynamic loads

\section{INTRODUCTION}

Since, the middle of the last decade, Graphene Platelets (GPLs) have been detected (Novoselov et al., 2004) and a wide range of studies have been achieved to discover the properties and performance of this new branch of advanced materials (Spitalsky et al., 2010; Geng et al., 2008; Li et al., 2008). Owing to their excellent and superior mechanical, electrical, thermal and magnetic properties as well as the lower cost production making them stellar choice as enhancement material in the composite structures (Debelak and Lafdi, 2007; Liao et al., 2008; Milani et al., 2013; Wang et al., 2015). As compared with the conventional composites which consume a high volume fraction of the carbon fibre (more than $60 \%$ ) it's found that by adding a small amount of weight fraction from the carbon filler to the resin matrix (about $0.1-5 \%$ ) lead to enormous increase in the mechanical properties of the nanocomposite (Putz et al., 2010). In the beginning, most of studies concentrated on the composition and the mechanical characterization of graphene nanocomposite.

Among of them, Rafiee et al. (2009) studied experimentally the buckling for composite beams reinforced with graphene and concluded that the adding of $0.1 \%$ of graphene leads to increase in the buckling load by $52 \%$ in comparison for the case of pure epoxy beam.
Also, Fang et al. (2009) fabricated graphene-polystyrene nanocomposite and detected that the adding of $0.9 \%$ from graphene leads to increase in the Young's modulus in about of $57 \%$. Wang et al. (2008) manufactured new nanocomposite through the adding graphene sheets with various sizes and their research presents that the utilizing for the large size of graphene sheets leads to great in increase in Young's modulus.

A multiscale modelling scheme used by Montazeri and Rafii-Tabar (2011) to study the mechanical characteristic of graphene-reinforced epoxy nanocomposite. Recently, several researches published on the structural behaviour of GPLs-reinforced composite. Chandra et al. (2012) analyzed free vibration of graphene-reinforced composite by utilizing the finite element method. Rissanou et al. (2015) performed the dynamic and structural analysis for graphene reinforced-nanocomposite by using different sizes of graphene sheets with using of the molecular dynamic simulation. Nowadays, the idea of Functionally Graded Materials (FGMs) to graphene reinforced the resin matrix nanocomposites inserted by Yang and his couthers (Yang et al., 2017) where the GPLs are non-uniformly scattered in the resin matrix. Song et al., $(2017 \mathrm{a}, \mathrm{b})$ investigated the linear free and forced vibration for the functionally graded material multi-layered graphene reinforced resin matrix composite plates.

Corresponding Author: Hamad M. Hasan, Department of Mechanical Engineering, College of Engineering, University of Anbar, Ramadi, Iraq 
They found that the weight fraction and the type of GPLs distribution have a great influence on the frequency and dynamic behaviour of the nanocomposite plates. Also, Song et al. $(2017 \mathrm{a}$, b) proposed a two-step perturbation technique to investigate the buckling and postbuckling of the functionally graded composite plates reinforced with multi-layer of graphene nanoplatelets under the effect of biaxial compression loads. Shen et al., (2017a, b) presents postbuckling of the composite plates reinforced by functionally graded graphene plates under the effect of thermal loads.

They concluded that the thermal postbuckling can be affected greatly with the functionally graded of graphene sheets. Also, Shen et al. (2017a, b) displayed the nonlinear vibration for the laminated composite plates reinforced by the functionally graded graphene sheets under the effect of thermal loads. The nonlinear frequencies determined by employing the two-step perturbation technique. It's found that the distribution kind of reinforcements and the thermal load has considerable effect on the nonlinear frequency. Indeed, the reinforcing the FG-Graphene circular cylindrical shells or cylindrical panels by stiffening members make them capable for carrying heavy dynamic loads in comparative with small addendum weight penalty.

Therefore, the nonlinear dynamic analysis of $\mathrm{FG}$ shells reinforced with eccentrically stiffeners received extensive studies from the researchers. Among those, Dung and Nam (2014) investigated the nonlinear dynamic behaviour of FG circular cylindrical shells on elastic foundation and reinforced with eccentrically stiffeners under the effect of external pressure. Bich et al. (2012) employed the classical Shelltheory together with the nonlinearity of the Von Karman to analyse the nonlinear dynamic behaviour of eccentrically stiffened FG cylindrical panels. Also, Bich et al. (2013) resolved the nonlinear dynamic and static buckling analysis of FG cylindrical thin shells reinforced by eccentrically stiffeners under the influence of axial compression loads. The response of the nonlinear imperfect FG circular cylindrical shells reinforced by eccentrically stiffeners on elastic foundation under the effect of comparison axial loads is studied by Duc and Thang (2014). Also, Duc and Quan (2013) considered the effect of the thermal loads on the nonlinear buckling of FG double curved shallow shells reinforced by external stiffeners on elastic foundation.

Finally, the nonlinear vibrations and dynamic behaviour for the piezoelectric FGM plates reinforced by eccentrically external stiffeners under the effect of mechanical and thermal loads investigated by Duc et al., (2016). It's clear that from the survey for the open literature, there are no publications on the nonlinear vibration and dynamic behaviour of eccentrically stiffened functionally graded graphene platelets-reinforced composite cylindrical panels. Due to the restrictions in the present industrialization technology, the manufacturing of an uninterruptedly through one dimensions is very tricky if not unattainable.

Therefore, multi-stratums structure containing of a number of individual stratums stacked up through which the weight fraction of the GPLs remains constant through each stratum but keep track of a layer-wise gradual change through the transverse direction is an excellent substantial if the overall number of stratums is adequately massive. The current research aims to search the nonlinear dynamic and vibration analysis of eccentrically stiffened reinforced with functionally graded GPLs-resin matrix composite cylindrical panels under the effect of dynamic load. The modified Halpin-Tsai method is utilized to determine the effective Young's modulus for the composite cylindrical panels and the stiffeners whereas the modified rule of mixture is employed to predict the poisson's ratio mass density.

Theoretical mathematical modelling is based on the classical theory. Galerkin method and Runge-Kutta scheme is used to determine the nonlinear frequencies as well as the dynamic behaviour of the simply supported composite cylindrical panels. A detailed parametric study is carried out to highlight the important insights for the influence of GPLs profile distribution, graphene weight fraction, stiffener geometric parameters, dynamic force and imperfection parameter on the nonlinear frequency as well as the dynamic behaviour of the functionally graded GPLs composite cylindrical panels.

Problem description: A cylindrical Shellpanel consist of a multi-layers GPLs-resin matrix with length $a$, width $b$, Radius R and thickness $h$ stiffened with eccentrically stiffeners under the effect of a uniform transverse dynamic load $\mathrm{Q}(\mathrm{x}, \mathrm{y}, \mathrm{t})$ is demonstrated in Fig. 1. The cylindrical panel consists of $\mathrm{N}$ stratum which has the same thickness and the GPLs uniformly scattered in the resin matrix through individual stratum.

The weight fraction of the GPLs shows a stratum-wise variation to compose a FG structure. With a view of examine the influence of GPLs distribution on the nonlinear frequency and the dynamic response, three kinds of FG material profile of GPLs, i.e., UD, FG_X and FG_O as shown in Fig. 2 may be investigated. The weight fraction of the GPLs in the first type UD is uniformly distributed at the same percent fraction across all the stratums. For the two types FG_X and FG_O, the weight fraction varies linearly from stratum to stratum across the panel thickness. The weight fraction decreases from the highest in the top and bottom to the lowest in the middle plane of the panel in the type FG_X whereas in the type FG_O, the case is reversed with the minimum weight fraction on the bottom and top planes and the highest in the middle plane of the panel. 


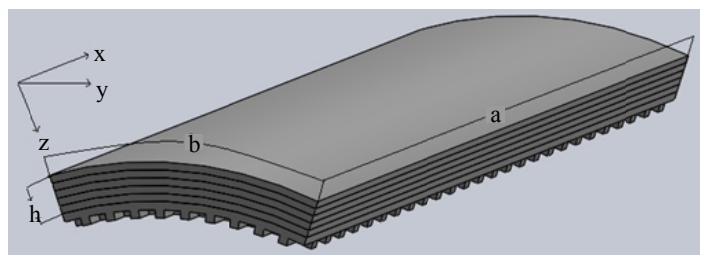

Fig. 1:Geometry of FG_GPLs eccentrically stiffened Shell panel

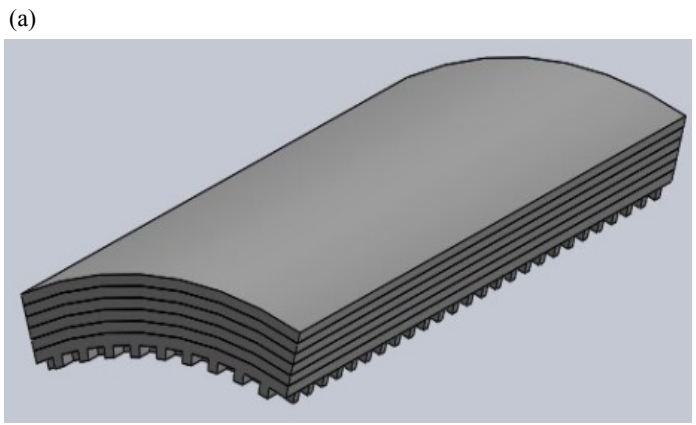

(b)

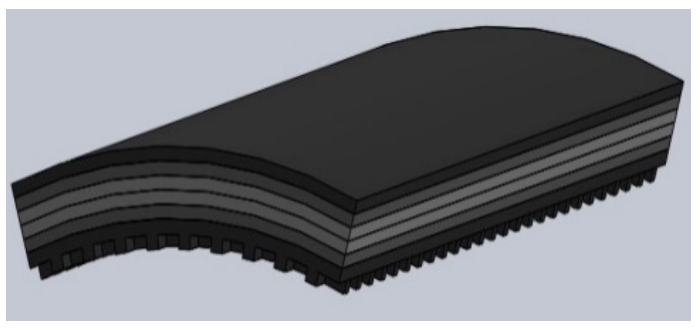

(c)

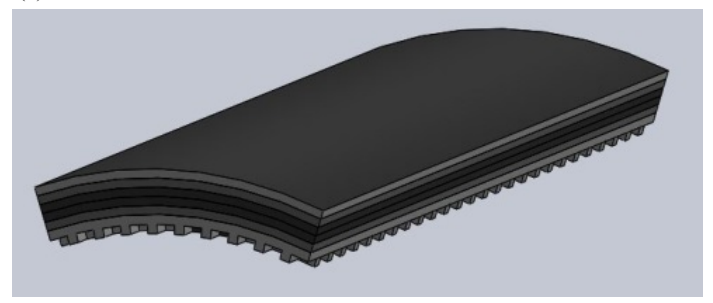

Fig. 2(a-c): Configuration of different GPLs Shellpanels (a) UD, (b) FG_X and (c) FG_O)

\section{MATERIALS AND METHODS}

Modified Halpin-Tsai method is adopted to determine the Young's modulus of the GPLs reinforced resin matrix of the composite panel. By considering GPLs are uniformly scattered in a resin matrix and the effective Young's modulus for the composite panel $\mathrm{E}$ can be estimated from the Voigt-Reuses method (Song et al., 2017a, b):

$$
\mathrm{E}_{\mathrm{C}}=\frac{3}{8} \mathrm{E}_{\mathrm{L}}+\frac{5}{8} \mathrm{E}_{\mathrm{T}}
$$

where, $\mathrm{E}_{\mathrm{L}}$ and $\mathrm{E}_{\mathrm{T}}$ are the longitudinal and transverse modulus, respectively and can be estimated by Halpin-Tsai method (Song et al., 2017a, b):

$$
\begin{gathered}
E_{L}=\frac{1+\xi_{L} \eta_{L} V_{G P L}}{1-\eta_{L} V_{G P L}} * E_{M} \\
E_{T}=\frac{1+\xi_{w} \eta_{w} V_{G P L}}{1-\eta_{W} V_{G P L}} * E_{M} \\
\eta_{L}=\frac{\left(E_{G P L} / E_{m}\right)-1}{\left(E_{G P L} / E_{m}\right)+\xi_{L}} \\
\eta_{W}=\frac{\left(E_{G P L} / E_{m}\right)-1}{\left(E_{G P L} / E_{m}\right)+\xi_{W}} \\
\xi_{L}=21_{G P L} / h_{G P L} \\
\xi_{W}=2 w_{G P L} / h_{G P L}
\end{gathered}
$$

Setting Eq. 2-7 into Eq. 1 gives:

$$
\begin{gathered}
\mathrm{E}_{\mathrm{C}}=\frac{3}{8} \frac{\left(\mathrm{E}_{\mathrm{GM}}+21_{\mathrm{GPL}} / \mathrm{h}_{\mathrm{GPL}}\right)+21_{\mathrm{GPL}} / h_{\mathrm{GPL}}\left(\mathrm{E}_{\mathrm{GM}}-1\right) \mathrm{V}_{\mathrm{GPL}}}{\mathrm{E}_{\mathrm{GM}}+21_{\mathrm{GPL}} / \mathrm{h}_{\mathrm{GPL}}-\left(\mathrm{E}_{\mathrm{GM}}-1\right) \mathrm{V}_{\mathrm{GPL}}} \mathrm{E}_{\mathrm{M}} \\
\frac{5}{8} \frac{\left(\mathrm{E}_{\mathrm{GM}}+2 \mathrm{w}_{\mathrm{GPL}} / \mathrm{h}_{\mathrm{GPL}}\right)+2 \mathrm{w}_{\mathrm{GPL}} / \mathrm{h}_{\mathrm{GPL}}\left(\mathrm{E}_{\mathrm{GM}}-1\right) \mathrm{V}_{\mathrm{GPL}}}{\mathrm{E}_{\mathrm{GM}}+2 \mathrm{w}_{\mathrm{GPL}} / \mathrm{h}_{\mathrm{GPL}}-\left(\mathrm{E}_{\mathrm{GM}}-1\right) \mathrm{V}_{\mathrm{GPL}}}
\end{gathered}
$$

where, $E_{\mathrm{GPI}}, 1_{\mathrm{GPL}} \mathrm{w}_{\mathrm{GPL}}$ and $\mathrm{h}_{\mathrm{GPL}}$ represent Young's modulus, length, width and thickness for GPLS, respectively while $\mathrm{E}_{\mathrm{M}}$ represents Young's modulus for the resin matrix:

$$
\mathrm{E}_{\mathrm{GM}}=\mathrm{E}_{\mathrm{GPL}} / \mathrm{E}_{\mathrm{M}}
$$

$\mathrm{V}_{\mathrm{GPL}}$ is the volume fraction of GPLs and can be estimated from:

$$
\mathrm{V}_{\mathrm{GPL}}=\frac{\mathrm{g}_{\mathrm{GPL}}}{\mathrm{g}_{\mathrm{GPL}}+\left(\rho_{\mathrm{GPL}} / \rho_{\mathrm{M}}\right)\left(1-\mathrm{g}_{\mathrm{GPL}}\right)}
$$

In which $\mathrm{g}_{\mathrm{GPL}}$ and $\rho_{\mathrm{GPL}}$ represent the weight fraction and mass density for GPLs, respectively while $\mathrm{p}_{M}$ is mass density for the resin matrix. The Poisson's ration $\mathrm{v}_{\mathrm{C}}$ and $\mathrm{p}_{\mathrm{C}}$ for the composite panel can be estimated from the rule of mixture:

$$
\begin{aligned}
& \mathrm{V}_{\mathrm{C}}=\mathrm{v}_{\mathrm{GPL}} \mathrm{V}_{\mathrm{GPL}}+\mathrm{v}_{\mathrm{M}} \mathrm{V}_{\mathrm{M}} \\
& \rho_{\mathrm{C}}=\rho_{\mathrm{GPL}} \mathrm{V}_{\mathrm{GPL}}+\rho_{\mathrm{M}} \mathrm{V}_{\mathrm{M}}
\end{aligned}
$$

Theoretical model for governing equations: The strains and curvatures at the midst surface of the functionally graded GPLs eccentrically stiffened cylindrical panels in 
accordance with the classical Shelltheory with Von Karman nonlinearity are related with the displacement components $\mathrm{u}, \mathrm{v}, \mathrm{w}$ in the $\mathrm{x}, \mathrm{y}, \mathrm{z}$ coordinates (Reddy and Liu, 1985):

$$
\begin{gathered}
\varepsilon_{x}^{0}=\frac{\partial u}{\partial x}+\frac{1}{2}\left(\frac{\partial w}{\partial x}\right)^{2}, k_{x}=-\frac{\partial^{2} w}{\partial x^{2}} \\
\varepsilon_{y}^{0}=\frac{\partial v}{\partial y}-\frac{1}{R} w+\frac{1}{2}\left(\frac{\partial w}{\partial y}\right)^{2}, k_{y}=-\frac{\partial^{2} w}{\partial y^{2}} \\
\gamma_{x y}^{0}=\frac{\partial u}{\partial y}+\frac{\partial v}{\partial x}+\frac{\partial w}{\partial x} \frac{\partial w}{\partial y}, k_{x y}=-\frac{\partial^{2} w}{\partial x \partial y}
\end{gathered}
$$

In which $\mathrm{R}$ is the radius of the cylindrical panel. The strains at any distance $z$ through the thickness of the panel are:

$$
\varepsilon_{\mathrm{x}}=\varepsilon_{\mathrm{x}}^{0}+\mathrm{zk}_{\mathrm{x}}, \varepsilon_{\mathrm{y}}=\varepsilon_{\mathrm{y}}^{0}+\mathrm{zk}_{\mathrm{y}}, \gamma_{\mathrm{xy}}=\gamma_{\mathrm{xy}}^{0}+2 \mathrm{zk}_{\mathrm{xy}}
$$

For a cylindrical shell, the geometric nonlinear compatibility equation:

$$
\frac{\partial^{2} \varepsilon_{x}^{0}}{\partial y^{2}}+\frac{\partial^{2} \varepsilon_{y}^{0}}{\partial x^{2}}-\frac{\partial^{2} \gamma_{x y}^{0}}{\partial x \partial y}=\left(\frac{\partial^{2} w}{\partial x \partial y}\right)^{2}-\frac{\partial^{2} w}{\partial x^{2}} \frac{\partial^{2} w}{\partial y^{2}}-\frac{1}{R} \frac{\partial^{2} w}{\partial x^{2}}
$$

The stress components for the Shellat the Nth layer can be evaluated from stress-strain relations:

$$
\begin{aligned}
& \sigma_{x}^{\operatorname{sh}(k)}=\frac{E_{\mathrm{c}}^{(k)}}{1-\mathrm{v}_{\mathrm{c}}^{2}} \varepsilon_{x}^{(k)}+\frac{\mathrm{vE}_{\mathrm{c}}^{(k)}}{1-\mathrm{v}_{\mathrm{c}}^{2}} \varepsilon_{\mathrm{y}}^{(k)} \\
& \sigma_{\mathrm{y}}^{\mathrm{sh}(\mathrm{k})}=\frac{E_{\mathrm{c}}^{(k)}}{1-\mathrm{v}_{\mathrm{c}}^{2}} \varepsilon_{\mathrm{y}}^{(k)}+\frac{\mathrm{vE}_{\mathrm{c}}^{(k)}}{1-\mathrm{v}_{\mathrm{c}}^{2}} \varepsilon_{\mathrm{x}}^{(k)} \\
& \sigma_{x y}^{\mathrm{sh}(\mathrm{k})}=\frac{E_{\mathrm{c}}^{(\mathrm{k})}}{2\left(1-\mathrm{V}_{\mathrm{c}}\right)} \gamma_{x y}^{(\mathrm{k})}
\end{aligned}
$$

And for the stiffeners (Bich and Long, 2010):

$$
\begin{aligned}
\sigma_{x}^{t} & =E_{0} \varepsilon_{x} \\
\sigma_{y}^{t} & =E_{0} \varepsilon_{y}
\end{aligned}
$$

In which $\mathrm{E}_{\circ}$ stands for elasticity modulus for the stiffeners and equal the value of elasticity modulus for the top and lower layer of the Shellpanel.

By taking into consideration the effect of stiffeners, the force and moment resultants can be obtained by integrating the stress-strains relations across the thickness of the panel (Najafizadeh et al., 2009):

$$
\begin{gathered}
N_{x}=\left(A_{11}+\frac{E_{0} A_{1}}{s_{1}}\right) \varepsilon_{x}^{0}+A_{12} \varepsilon_{y}^{0}-\left(B_{11}+C_{1}\right) k_{x}-B_{12} k_{y} \\
N_{y}=A_{12} \varepsilon_{x}^{0}+\left(A_{22} \frac{E_{0} A_{2}}{s_{2}}\right) \varepsilon_{y}^{0}-B_{12} k_{x}-\left(B_{22}+C_{2}\right) k_{y} \\
N_{x y}=A_{6 \gamma} \gamma_{x y}^{0}-2 B_{66} k_{x y} \\
M_{x}=\left(B_{11}+C_{1}\right) \varepsilon_{x}^{0}+B_{12} \varepsilon_{y}^{0}-\left(D_{11}+\frac{E_{0} 1_{1}}{s_{1}}\right) k_{x}-D_{12} k_{y} \\
M_{y}=B_{12} \varepsilon_{x}^{0}+\left(B_{22}+C_{2}\right) \varepsilon_{y}^{0}-D_{12} k_{x}-\left(D_{22}+\frac{E_{0} 1_{2}}{s_{2}}\right) k_{y} \\
M_{x y}=B_{66} \gamma_{x y}^{0}-2 D_{66} \gamma_{x y}
\end{gathered}
$$

In the above relations $A_{i j}, B_{i j}$ and $D_{i j}$ are the Shellstiffness's without stiffeners and represented by:

$$
\begin{aligned}
& A_{11}=A_{22}=\sum_{k=1}^{N} \int_{z_{k}}^{a_{k-1}} \frac{E_{c}^{(k)}(z)}{1-v_{c}^{2}} d z, A_{12}=\sum_{k=1}^{N} \int_{z_{k}}^{k_{k} \cdot 1} \frac{E_{c}^{(k)}(z)}{1-v_{c}^{2}} d z \\
& A_{66}=\sum_{k=1}^{N} \int_{z_{k}}^{z_{k} \cdot 1} \frac{E_{c}^{(k)}(z)}{2\left(1+v_{c}\right)} d z
\end{aligned}
$$

$$
\begin{aligned}
& \mathrm{B}_{11}=\mathrm{B}_{22}=\sum_{\mathrm{k}=1}^{\mathrm{N}} \int_{z_{\mathrm{k}}}^{z_{\mathrm{k}_{\mathrm{k}} \cdot}} \frac{\mathrm{E}_{\mathrm{c}}^{(\mathrm{k})}(\mathrm{z})}{2\left(1+\mathrm{v}_{\mathrm{c}}^{2}\right)} \mathrm{dz}, \mathrm{B}_{12}=\sum_{\mathrm{k}=1}^{\mathrm{N}} \int_{z_{\mathrm{k}}}^{z_{\mathrm{k} \cdot \mathrm{k}}} \frac{\mathrm{vE}_{\mathrm{c}}^{(\mathrm{k})}(\mathrm{z})}{\left(1-\mathrm{v}_{\mathrm{c}}^{2}\right)} \mathrm{dz} \\
& \mathrm{B}_{66}=\sum_{\mathrm{k}=1}^{\mathrm{N}} \int_{z_{\mathrm{k}}}^{\mathrm{z}_{\mathrm{k} \cdot 1}} \frac{\mathrm{E}_{\mathrm{c}}^{(\mathrm{k})}(\mathrm{z})}{2\left(1+\mathrm{v}_{\mathrm{c}}\right)} \mathrm{dz} \\
& D_{11}=D_{22}=\sum_{k=1}^{N} \int_{z_{k}}^{z_{k-1}} \frac{E_{c}^{(k)}(z)}{2\left(1+v_{c}^{2}\right)} z^{2} d z \\
& D_{12}=\sum_{k=1}^{N} \int_{z_{k}}^{z_{k} \cdot 1} \frac{E_{c}^{(k)}(z)}{2\left(1-v_{c}^{2}\right)} z^{2} d z D_{66}=\sum_{k=1}^{N} \int_{z_{z_{k}}}^{z_{k n}} \frac{E_{c}^{(k)}(z)}{2\left(1+v_{c}\right)} z^{2} d z
\end{aligned}
$$

Also, the relations for stiffeners geometric parameters can be represented by:

$$
\begin{gathered}
\mathrm{l}_{1}=\frac{\mathrm{d}_{1} \mathrm{~h}_{1}^{3}}{12}+\mathrm{A}_{1} \mathrm{z}_{1}^{2}, \mathrm{l}_{2}=\frac{\mathrm{d}_{2} \mathrm{~h}_{2}^{3}}{12}+\mathrm{A}_{2} \mathrm{z}_{2}^{2} \\
\mathrm{C}_{1}=\frac{\mathrm{E}_{\mathrm{C}} \mathrm{A}_{1} \mathrm{z}_{1}}{\mathrm{~s}_{1}}, \mathrm{C}_{2}=\frac{\mathrm{E}_{\mathrm{C}} \mathrm{A}_{2} \mathrm{z}_{2}}{\mathrm{~s}_{2}} \\
\mathrm{z}_{1}=\frac{\mathrm{h}_{1}+\mathrm{h}}{2}, \mathrm{z}_{2}=\frac{\mathrm{h}_{2}+\mathrm{h}}{2}
\end{gathered}
$$


The strains in terms of the force resultants can be obtained from Eq. 16:

In which:

$$
\begin{gathered}
\varepsilon_{\mathrm{x}}^{\circ}=\mathrm{E}_{22} \mathrm{~N}_{\mathrm{x}}-\mathrm{E}_{12} \mathrm{~N}_{\mathrm{y}}+\mathrm{G}_{11} \mathrm{k}_{\mathrm{x}}+\mathrm{G}_{12} \mathrm{k}_{\mathrm{y}} \\
\varepsilon_{\mathrm{y}}^{\circ}=\mathrm{E}_{11} \mathrm{~N}_{\mathrm{y}}-\mathrm{E}_{12} \mathrm{~N}_{\mathrm{x}}+\mathrm{G}_{21} \mathrm{k}_{\mathrm{x}}+\mathrm{G}_{22} \mathrm{k}_{\mathrm{y}} \\
\gamma_{\mathrm{xy}}^{\circ}=\mathrm{E}_{66}+2 \mathrm{G}_{66} \mathrm{k}_{\mathrm{xy}}
\end{gathered}
$$

$$
\begin{gathered}
\mathrm{E}_{11}=\frac{1}{\Lambda}\left(\mathrm{A}_{11}+\frac{\mathrm{E}_{\mathrm{c}} \mathrm{A}_{1}}{\mathrm{~s}_{1}}\right), \mathrm{E}_{22}=\frac{1}{\Lambda}\left(\mathrm{A}_{22}+\frac{\mathrm{E}_{\mathrm{c}} \mathrm{A}_{2}}{\mathrm{~s}_{2}}\right) \\
\mathrm{E}_{12}=\frac{\mathrm{A}_{22}}{\Lambda}, \mathrm{E}_{66}=\frac{1}{\mathrm{~A}_{66}}, \Lambda=\left(\mathrm{A}_{11}+\frac{\mathrm{E}_{\mathrm{c}} \mathrm{A}}{\mathrm{s}_{1}}\right)\left(\mathrm{A}_{22}+\frac{\mathrm{E}_{\mathrm{c}} \mathrm{A}_{2}}{\mathrm{~s}_{2}}\right)-\mathrm{A}_{12}^{2} \\
\mathrm{G}_{11}=\mathrm{E}_{22}\left(\mathrm{~B}_{11}+\mathrm{C}_{1}\right)-\mathrm{E}_{12} \mathrm{~B}_{12}, \mathrm{G}_{22}=\mathrm{E}_{11}\left(\mathrm{~B}_{22}+\mathrm{C}_{2}\right)-\mathrm{E}_{12} \mathrm{~B}_{12} \\
\mathrm{G}_{12}=\mathrm{E}_{22} \mathrm{~B}_{12}-\mathrm{E}_{12}\left(\mathrm{~B}_{22}+\mathrm{C}_{2}\right), \mathrm{G}_{21}=\mathrm{E}_{11} \mathrm{~B}_{12}-\mathrm{E}_{12}\left(\mathrm{~B}_{11}+\mathrm{C}_{1}\right) \\
\mathrm{G}_{66}=\frac{\mathrm{B}_{66}}{\mathrm{~A}_{66}}
\end{gathered}
$$

By substituting Eq. 18 into Eq. 15 gives:

$$
\begin{gathered}
M_{\mathrm{x}}=\mathrm{G}_{11} \mathrm{~N}_{\mathrm{x}}+\mathrm{G}_{21} \mathrm{~N}_{\mathrm{y}} \mathrm{F}_{11} \mathrm{k}_{\mathrm{x}}-\mathrm{F}_{12} \mathrm{k}_{\mathrm{y}} \\
\mathrm{M}_{\mathrm{y}}=\mathrm{G}_{12} \mathrm{~N}_{\mathrm{x}}+\mathrm{G}_{22} \mathrm{~N}_{\mathrm{y}}-\mathrm{F}_{21} \mathrm{k}_{\mathrm{x}}-\mathrm{F}_{22} \mathrm{k}_{\mathrm{y}} \\
\mathrm{M}_{\mathrm{xy}}=\mathrm{G}_{66} \mathrm{~N}_{\mathrm{xy}}-2 \mathrm{~F}_{66} \mathrm{~N}_{\mathrm{xy}}
\end{gathered}
$$

In which:

$$
\begin{gathered}
\mathrm{F}_{11}=\mathrm{D}_{11}+\frac{\mathrm{E}_{\mathrm{c}} \mathrm{I}_{1}}{\mathrm{~s}_{1}}-\left(\mathrm{B}_{11}+\mathrm{C}_{1}\right) \mathrm{G}_{11}-\mathrm{G}_{12} \mathrm{G}_{21} \\
\mathrm{~F}_{22}=\mathrm{D}_{22}+\frac{\mathrm{E}_{\mathrm{c}} \mathrm{I}_{2}}{\mathrm{~s}_{1}}-\left(\mathrm{B}_{22}+\mathrm{C}_{2}\right) \mathrm{G}_{22}-\mathrm{B}_{12} \mathrm{G}_{22} \\
\mathrm{~F}_{12}=\mathrm{D}_{12}-\left(\mathrm{B}_{11}+\mathrm{C}_{1}\right) \mathrm{G}_{12}-\mathrm{B}_{12} \mathrm{G}_{22} \\
\mathrm{~F}_{21}=\mathrm{D}_{12}-\mathrm{B}_{12} \mathrm{G}_{11}-\left(\mathrm{B}_{22}+\mathrm{C}_{2}\right) \mathrm{G}_{21} \\
\mathrm{~F}_{66}=\mathrm{D}_{66}-\mathrm{B}_{66} \mathrm{G}_{66}
\end{gathered}
$$

In accordance with the classical Shelltheory, the nonlinear equations of motion are:

$$
\begin{aligned}
& \frac{\partial N x}{\partial x}+\frac{\partial N x y}{\partial y}=0 \\
& \frac{\partial N x y}{\partial x}+\frac{\partial N y}{\partial y}=0
\end{aligned}
$$

$$
\begin{gathered}
\frac{\partial^{2} \mathrm{Mx}}{\partial \mathrm{x}^{2}}+2 \frac{\partial^{2} \mathrm{Mxy}}{\partial \mathrm{x} y}+\frac{\partial^{2} \mathrm{My}}{\partial \mathrm{y}^{2}}+\mathrm{Nx} \frac{\partial^{2} \mathrm{w}}{\partial \mathrm{x}^{2}}+ \\
2 \mathrm{Nxy} \frac{\partial^{2} \mathrm{w}}{\partial \mathrm{x} \partial \mathrm{y}}+\mathrm{Ny} \frac{\partial^{2} \mathrm{w}}{\partial \mathrm{y}^{2}}+\frac{1}{\mathrm{R}} \mathrm{Ny}+\mathrm{q}_{\mathrm{o}}=\mathrm{I}_{1}^{+} \frac{\partial^{2} \mathrm{w}}{\partial \mathrm{t}^{2}} \\
\mathrm{I}_{1}^{*}=\sum_{\mathrm{k}=1}^{\mathrm{N}} \int_{\mathrm{z}_{\mathrm{k}}}^{z_{\mathrm{k} \cdot \mathrm{k}}} \rho_{\mathrm{c}}(\mathrm{z}) \mathrm{dz}+\rho_{\mathrm{o}}\left(\frac{\mathrm{A}_{1}}{\mathrm{~s}_{1}}+\frac{\mathrm{A}_{2}}{\mathrm{~s}_{2}}\right)
\end{gathered}
$$

In which:

Where:

$\rho_{c} \quad$ : The mass density for the composite panel

$\rho_{0}$ : The mass density for the stiffeners and takes the same value of mass density for the top layer of the composite panel

The stress function:

$$
N x=\frac{\partial^{2} f}{\partial y^{2}}, N y=\frac{\partial^{2} f}{\partial x^{2}}, N x y=-\frac{\partial^{2} f}{\partial x \partial y}
$$

The inserting of Eq. 19 into Eq. 14 and 21 into Eq. 23 with taken into consideration Eq. 12 and 24 gives a system of nonlinear equations:

$$
\begin{gathered}
\mathrm{E}_{11} \frac{\partial^{4} \mathrm{f}}{\partial \mathrm{x}^{4}}+\left(\mathrm{E}_{66}-2 \mathrm{E}_{12}\right) \frac{\partial^{4} \mathrm{f}}{\partial \mathrm{x}^{2} \partial \mathrm{y}^{2}}+\mathrm{E}_{22} \frac{\partial^{4} \mathrm{f}}{\partial \mathrm{y}^{4}}+\mathrm{G}_{21} \frac{\partial^{4} \mathrm{w}}{\partial \mathrm{x}^{4}}+ \\
\left(\mathrm{G}_{11}+\mathrm{G}_{22}-2 \mathrm{G}_{66}\right) \frac{\partial^{4} \mathrm{w}}{\partial \mathrm{x}^{2} \partial \mathrm{y}^{2}}+\mathrm{G}_{12} \frac{\partial^{4} \mathrm{w}}{\partial \mathrm{y}^{4}}+\frac{1}{\mathrm{R}} \frac{\partial^{2} \mathrm{w}}{\partial \mathrm{x}^{2}}= \\
\left(\frac{\partial^{2} \mathrm{w}}{\partial \mathrm{x} \partial \mathrm{y}}\right)^{2} \frac{\partial^{2} \mathrm{w}}{\partial \mathrm{x}^{2}} \frac{\partial^{2} \mathrm{w}}{\partial \mathrm{y}^{2}} \\
\mathrm{I}_{1}^{*} \frac{\partial^{2} \mathrm{w}}{\partial \mathrm{t}^{2}}+\mathrm{F}_{11} \frac{\partial^{4} \mathrm{w}}{\partial \mathrm{x}^{4}}+\left(\mathrm{F}_{12}+\mathrm{F}_{21}+4 \mathrm{~F}_{66}\right) \frac{\partial^{4} \mathrm{w}}{\partial \mathrm{x}^{2} \partial \mathrm{y}^{2}}+\mathrm{F}_{22} \frac{\partial^{4} \mathrm{w}}{\partial \mathrm{y}^{4}}-\mathrm{G}_{21} \frac{\partial^{4} \mathrm{f}}{\partial \mathrm{x}^{4}}- \\
\left(\mathrm{G}_{11}+\mathrm{G}_{22}-2 \mathrm{G}_{66}\right) \frac{\partial^{4} \mathrm{f}}{\partial \mathrm{x}^{2} \partial \mathrm{y}^{2}}-\mathrm{G}_{12} \frac{\partial^{4} \mathrm{f}}{\partial \mathrm{y}^{4}}-\frac{1}{\mathrm{R}} \frac{\partial^{2} \mathrm{f}}{\partial \mathrm{x}^{2}}-\frac{\partial^{2} \mathrm{f}}{\partial \mathrm{y}^{2}} \frac{\partial^{2} \mathrm{w}}{\partial \mathrm{x}^{2}}+ \\
2 \frac{\partial^{2} \mathrm{f}}{\partial \mathrm{x} \partial \mathrm{y}} \frac{\partial^{2} \mathrm{w}}{\partial \mathrm{x} \partial \mathrm{y}}-\frac{\partial^{2} \mathrm{f}}{\partial \mathrm{x}^{2}} \frac{\partial^{2} \mathrm{w}}{\partial \mathrm{y}^{2}}=\mathrm{q}_{\circ}
\end{gathered}
$$

For taking into consideration, the initial imperfection for the FG-GPLs cylindrical panels, $\mathrm{w}_{\circ}$ represent a known very small imperfection and as in the Volmir's Model (Shen, 2001) for an imperfection cylindrical panels, the equation of motion for an imperfect FG-GPLs stiffened cylindrical panels becomes:

$$
\begin{aligned}
& \mathrm{E}_{11} \frac{\partial^{4} \mathrm{f}}{\partial \mathrm{x}^{4}}+\left(\mathrm{E}_{66}-2 \mathrm{~F}_{12}\right) \frac{\partial^{4} \mathrm{f}}{\partial \mathrm{x}^{2} \partial \mathrm{y}^{2}}+\mathrm{E}_{22} \frac{\partial^{4} \mathrm{f}}{\partial \mathrm{y}^{4}}+\mathrm{G}_{21} \frac{\partial^{4}\left(\mathrm{w}-\mathrm{w}_{\mathrm{o}}\right)}{\partial \mathrm{x}^{4}}+ \\
& \left(\mathrm{G}_{11}+\mathrm{G}_{22}-2 \mathrm{G}_{66}\right) \frac{\partial^{4}\left(\mathrm{w}-\mathrm{w}_{\mathrm{o}}\right)}{\partial \mathrm{x}^{2} \mathrm{y}^{2}}+\mathrm{G}_{12} \frac{\partial^{4}\left(\mathrm{w}-\mathrm{w}_{\mathrm{o}}\right)}{\partial \mathrm{y}^{4}}+\frac{1}{\mathrm{R}} \\
& \frac{\partial^{2}\left(\mathrm{w}-\mathrm{W}_{\mathrm{o}}\right)}{\partial \mathrm{x}^{2}}=\left[\left(\frac{\partial^{2} \mathrm{w}}{\partial \mathrm{x} \partial \mathrm{y}}\right)^{2} \frac{\partial^{2} \mathrm{w} \partial^{2} \mathrm{w}}{\partial \mathrm{x}^{2} \partial \mathrm{y}^{2}}\right]-\left[\left(\frac{\partial^{2} \mathrm{w}_{\mathrm{o}}}{\partial \mathrm{x} \partial \mathrm{y}}\right)^{2} \frac{\partial^{2} \mathrm{w}_{\mathrm{o}}}{\partial \mathrm{x}^{2}} \frac{\partial^{2} \mathrm{w}_{\mathrm{o}}}{\partial \mathrm{y}^{2}}\right]
\end{aligned}
$$




$$
\begin{aligned}
& \mathrm{I}_{1}^{*} \frac{\partial^{2} \mathrm{~W}}{\partial \mathrm{t}^{2}}+\mathrm{F}_{11} \frac{\partial^{4}\left(\mathrm{~W}-\mathrm{W}_{\mathrm{o}}\right)}{\partial \mathrm{x}^{4}}+\left(\mathrm{F}_{12}+\mathrm{F}_{21}+4 \mathrm{~F}_{66}\right) \frac{\partial^{4}\left(\mathrm{w}-\mathrm{w}_{\mathrm{o}}\right)}{\partial \mathrm{x}^{2} \partial \mathrm{y}^{2}}+ \\
& \mathrm{F}_{22} \frac{\partial^{4}\left(\mathrm{w}-\mathrm{w}_{0}\right)}{\partial \mathrm{y}^{4}}-\mathrm{G}_{21} \frac{\partial^{4} \mathrm{f}}{\partial \mathrm{x}^{4}}-\left(\mathrm{G}_{11}+\mathrm{G}_{22}-2 \mathrm{G}_{66}\right) \frac{\partial^{4} \mathrm{f}}{\partial \mathrm{x}^{2} \partial \mathrm{y}^{2}}-\mathrm{G}_{12} \frac{\partial^{4} \mathrm{f}}{\partial \mathrm{y}^{4}}(28) \\
& \frac{1}{\mathrm{R}} \frac{\partial^{2} \mathrm{f}}{\partial \mathrm{x}^{2}} \frac{\partial^{2} \mathrm{f}}{\partial \mathrm{y}^{2}} \frac{\partial^{2} \mathrm{w}}{\partial \mathrm{x}^{2}}+2 \frac{\partial^{2} \mathrm{f}}{\partial \mathrm{x} \partial \mathrm{y}} \frac{\partial^{2} \mathrm{~W}}{\partial \mathrm{x} \partial \mathrm{y}} \frac{\partial^{2} \mathrm{f}}{\partial \mathrm{x}^{2}} \frac{\partial^{2} \mathrm{w}}{\partial \mathrm{y}^{2}}=\mathrm{q}_{\circ}
\end{aligned}
$$

In which $w$ represent the deflection of the cylindrical panel. Equation 27 and 28 are nonlinear equations and can be employed to study the vibration and dynamic behaviour for imperfection FG-GPLs eccentrically stiffened cylindrical panels.

Problem solution: By assuming an imperfect FG-GPLs eccentrically stiffened cylindrical panel is simply supported under the effect of uniformly distributed pressure $\mathrm{q}_{0}$. So, for the present case study, the boundary conditions:

$$
\begin{aligned}
& \mathrm{w}=0, \mathrm{Mx}=0, \mathrm{Nx}=0, \mathrm{Nxy}=0 \text { at } \mathrm{x}=0, \mathrm{x}=\mathrm{a} \\
& \mathrm{w}=0, \mathrm{My}=0, \mathrm{Ny}=0, \mathrm{Nxy}=0 \text { at } \mathrm{y}=0, \mathrm{y}=\mathrm{a}
\end{aligned}
$$

The above boundary conditions can be satisfied if the solution for the panel deflection in the form as:

$$
w=w(t) \sin \frac{m \pi x}{a} \sin \frac{n \pi y}{b}
$$

In which $w(t)$ is function of time and represent the amplitude, $m, n$ represent the half wave number in the longitudinal and circumferential directions. For the case of initial imperfection, it's consider identically to the solution for the panel deflection:

$$
w_{o}=w_{0} \sin \frac{m \pi x}{a} \sin \frac{n \pi y}{b}
$$

By solving Eq. 30 and 31 with Eq. 14 and by defining the stress function as:

$$
\begin{aligned}
& f(x, y, t)=A_{1}(t) \cos \frac{2 m \pi x}{a}+A_{2}(t) \cos \frac{2 n \pi y}{b}- \\
& A_{3}(t) \sin \frac{m \pi x}{a} \sin \frac{n \pi y}{b}
\end{aligned}
$$

In which:

$$
\begin{gathered}
A_{1}=\frac{n^{2}(a / b)^{2} w^{2}}{32 m^{2} E_{11}}, A_{2}=\frac{m^{2} w^{2}}{32 n^{2}(a / b)^{2} E_{22}}, \\
A_{1}=\frac{n^{2}(a / b)^{2} w^{2}}{32 m^{2} E_{11}}, A_{2}=\frac{m^{2} w^{2}}{32 n^{2}(a / b)^{2} E_{22}}, A_{3}= \\
\frac{\left[G_{21} m^{4}+\left(G_{11}+G_{22}-2 G_{66}\right) m^{2} n^{2}(a / b)^{2}+G_{12} n^{4}(a / b)^{4}-\left(a / \pi^{2}\right)(1 / R) m^{2}\right.}{E_{11} m^{4}+\left(E_{66}-2 E_{12}\right) m^{2} n^{2}(a / b)^{2}+E_{22} n^{4}(a / b)^{4}}
\end{gathered}
$$

By substituting the relations (30-32) into Eq. 26 and then by applying Galerk in approach, it gives:

$$
\begin{aligned}
& a_{1} w^{\prime \prime}+\left(a_{2}+\frac{a_{3}^{2}}{a_{4}}\right)\left(w-w_{0}\right)+\frac{32 m n(a / b)^{2}}{3 \pi} \frac{a_{3}}{a_{4}}\left(w-w_{0}\right) w+ \\
& a_{5}\left(w^{2}-w_{0}^{2}\right)+a_{6}\left(w^{2}-w_{0}^{2}\right) w-\frac{16 a^{4} q_{0}}{m n \pi^{6}}=0
\end{aligned}
$$

In which:

$$
\begin{aligned}
& \mathrm{a}_{1}=\frac{\mathrm{a}^{4}}{\pi^{4}} \mathrm{I}_{1}^{*}, \mathrm{a}_{2}=\mathrm{m}^{4} \mathrm{~F}_{11}+\left(\mathrm{F}_{12}+\mathrm{F}_{21}+4 \mathrm{~F}_{66}\right) \mathrm{m}^{2} \mathrm{n}^{2}(\mathrm{a} / \mathrm{b})^{2}+\mathrm{F}_{22} \mathrm{n}^{4}(\mathrm{a} / \mathrm{b})^{4} \\
& \mathrm{a}_{3}=\mathrm{G}_{21} \mathrm{~m}^{4}+\left(\mathrm{G}_{11}+\mathrm{G}_{22}-2 \mathrm{G}_{66}\right) \mathrm{m}^{2} \mathrm{n}^{2}(\mathrm{a} / \mathrm{b})^{2}+\mathrm{G}_{12} \mathrm{n}^{4}(\mathrm{a} / \mathrm{b})^{4}-\frac{\mathrm{a}^{2}}{\pi^{2}} \frac{1}{\mathrm{R}} \mathrm{m}^{2} \\
& \mathrm{a}_{4}=\mathrm{E}_{11} \mathrm{~m}^{4}+\left(\mathrm{E}_{66}-2 \mathrm{E}_{12}\right) \mathrm{m}^{2} \mathrm{n}^{2}(\mathrm{a} / \mathrm{b})^{2}+\mathrm{E}_{22} \mathrm{n}^{4}(\mathrm{a} / \mathrm{b})^{4} \\
& \mathrm{a}_{5}=\frac{8 \mathrm{mn}(\mathrm{a} / \mathrm{b})^{2}}{3 \pi^{2}}\left(\frac{\mathrm{G}_{21}}{\mathrm{E}_{11}}+\frac{\mathrm{G}_{12}}{\mathrm{E}_{22}}\right)-\left(\frac{8 \mathrm{a}^{2}}{6 \pi^{4} \mathrm{mn}} \frac{\mathrm{n}^{2}(\mathrm{a} / \mathrm{b})^{2}}{\mathrm{E}_{11}} \frac{1}{\mathrm{R}}\right) \\
& \mathrm{a}_{6}=\frac{1}{16}\left(\frac{\mathrm{m}^{4}}{\mathrm{E}_{22}}+\frac{\mathrm{n}^{4}(\mathrm{a} / \mathrm{b})^{4}}{\mathrm{E}_{11}}\right)
\end{aligned}
$$

By considering the uniform distributed load $\mathrm{q}_{\mathrm{o}}=\mathrm{Q}$ $\sin \Omega$ t Eq. 34 becomes:

$$
\begin{aligned}
& \mathrm{a}_{1} \mathrm{w}^{\prime \prime}+\left(\mathrm{a}_{2}+\frac{\mathrm{a}_{3}^{2}}{\mathrm{a}_{4}}\right)\left(\mathrm{w}-\mathrm{w}_{\mathrm{o}}\right)+\frac{32 \mathrm{mn}(\mathrm{a} / \mathrm{b})^{2}}{3 \pi} \frac{\mathrm{a}_{3}}{\mathrm{a}_{4}}\left(\mathrm{w}-\mathrm{w}_{\mathrm{o}}\right) \\
& \mathrm{w}+\mathrm{a}_{5}\left(\mathrm{w}^{2}-\mathrm{w}_{\mathrm{o}}^{2}\right)+\mathrm{a}_{6}\left(\mathrm{w}^{2}-\mathrm{w}_{\mathrm{o}}^{2}\right) \mathrm{w}=\frac{16 \mathrm{a}^{4}}{\mathrm{mn} \pi^{6}} \mathrm{Q} \sin \Omega \mathrm{t}
\end{aligned}
$$

Thus, the nonlinear dynamic behaviour for the FG-GPLs imperfection stiffened cylindrical panels can be determined by resolving the above equation in combination with the initial conditions to be considered as $\mathrm{w}(0)=0, \mathrm{w}^{*}(0)=0$, by employing the Runge-Kutta method. For linear and free vibration, Eq. 36 becomes:

$$
a_{1} w^{\prime \prime}+\left(a_{2}+\frac{a_{3}^{2}}{a_{4}}\right)\left(w-w_{0}\right)=0
$$

From the above equation, the fundamental frequencies for the natural vibration of FG-GPLs imperfection stiffened panel can be obtained as:

$$
\omega_{L}=\sqrt{\frac{1}{a_{1}}\left(a_{2}+\frac{a_{3}^{2}}{a^{4}}\right)}
$$

Also, for nonlinear free vibration, Eq. 36 with perfect panel can be written as:

$$
\begin{gathered}
w^{\prime \prime}+n_{1} w+n_{1} w^{2}+n_{3} w^{3}=0 \\
n_{1}=\frac{1}{a_{1}}\left(a_{2}+\frac{a_{3}^{2}}{a_{4}}\right), n_{2}=\frac{1}{a_{1}}\left(\frac{32 m n(a / b)^{2}}{3 \pi^{2}} \frac{a_{3}}{a_{4}}+a_{5}\right), n_{3}=\frac{a_{6}}{a_{1}}
\end{gathered}
$$


Let $w(t)=X \sin \Omega t$ and by applying Galerkin method to Eq. 39, the frequency-amplitude relation can be determined:

$$
\frac{\omega_{\mathrm{NL}}}{\omega_{\mathrm{L}}}=\left(1+\frac{8 \mathrm{n}_{2}}{3 \pi \omega_{\mathrm{L}}^{2}} \mathrm{X}+\frac{3 \mathrm{n}_{3}}{4 \omega_{\mathrm{L}}^{2}} \mathrm{X}^{2}\right)^{\frac{1}{2}}
$$

Where:

$\omega_{\mathrm{NL}}$ : The nonlinear vibration frequency

$\mathrm{X}$ : The amplitude of nonlinear vibration

\section{RESULTS AND DISCUSSION}

Validation of the current case study: In this portion, to prove validation the present case approach, the fundamental frequency change with the weight fraction of GPLs for multi-stratums FG-GPLs reinforcing epoxy matrix plates are compared in Fig. 3 with the results obtained in Song et al. $(2017 \mathrm{a}, \mathrm{b})$. The variations in the fundamental frequencies are represented for different GPLs distribution patterns. This comparison reveals that the outcomes from the current approach are in a good acceptance with the published results.

Vibration results: In order to explain the suggested analysis for multi-stratums FG-GPLs reinforced with external stiffeners thin cylindrical panels $(\mathrm{a} \times \mathrm{b} \times \mathrm{h}=$ $1.5 \times 1.5 \times 0.008 \mathrm{~m}$ ), the dimensions and material properties for GPLs and resin matrix are:

$$
\begin{aligned}
& 1_{\mathrm{GPL}}=2.5 \mu \mathrm{m}, \quad \mathrm{w}_{\mathrm{GPL}}=1.5 \mu \mathrm{m}, \quad \mathrm{h}_{\mathrm{GPL}}=1.5 \mathrm{~nm}, \\
& \rho_{\mathrm{GPL}}=1.06 \mathrm{~g} \mathrm{~cm}^{-3}, \quad \mathrm{E}_{\mathrm{GPL}}=1.01 \mathrm{Tpa}, \rho_{\mathrm{m}}=1.2 \mathrm{~g} \mathrm{~cm}^{-3}, \\
& \mathrm{E}_{\mathrm{M}}=3.0 \mathrm{Gpa}, \quad \mathrm{v}_{\mathrm{m}}=0.34, \quad \mathrm{v}_{\mathrm{GPL}}=0.186
\end{aligned}
$$

In addition, the dimensions for geometric parameters of stiffeners:

$$
\mathrm{h}_{1}=\mathrm{h}_{2}=0.015 \mathrm{~m}, \mathrm{~s}_{1}=\mathrm{s}_{2}=0.015 \mathrm{~m}, \mathrm{~d}_{1}=\mathrm{d}_{2}=0.04 \mathrm{~m}
$$

Results of natural vibrations: The results taken out in Table 1 exhibit that the influence of stiffeners on the fundamental frequencies for the natural vibration $\left(\omega_{L}\right)$ is significant. It's clearly that the fundamental frequencies for FG-GPLs panels reinforcing with stiffeners or without stiffeners to be relied mainly on the weight fraction and distribution style of GPLs. It is evident that the percentage increase in $\omega_{\mathrm{L}}$ is $105.9 \%$ when the weight fraction of GPLs increase from $1-5 \%$ in the case of FG_X distribution pattern. Also in the case of FG_X and FG_O distribution pattern, the percentage increase in the natural frequencies is $47.4 \%$.

Results of nonlinear frequency-amplitude free vibration: Figure 4 displays the effect of weight fraction on the nonlinear frequency for different GPLs distribution

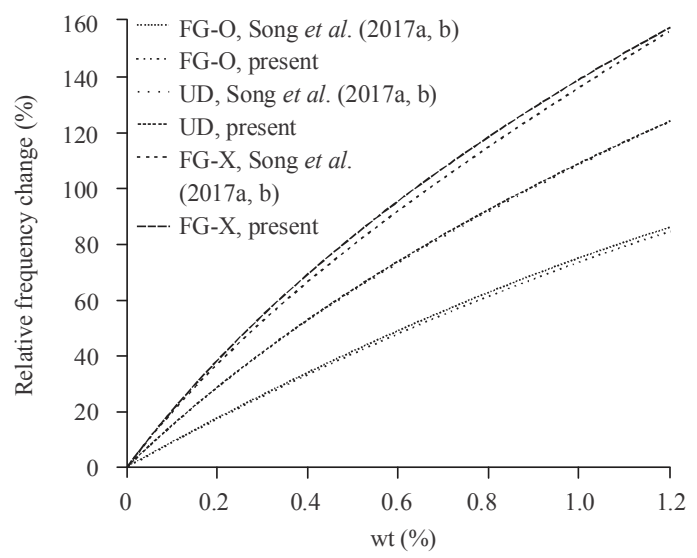

Fig. 3: Comparison of relative frequency change-weight fraction with results published by Song et al. (2017a, b) for different distribution patterns

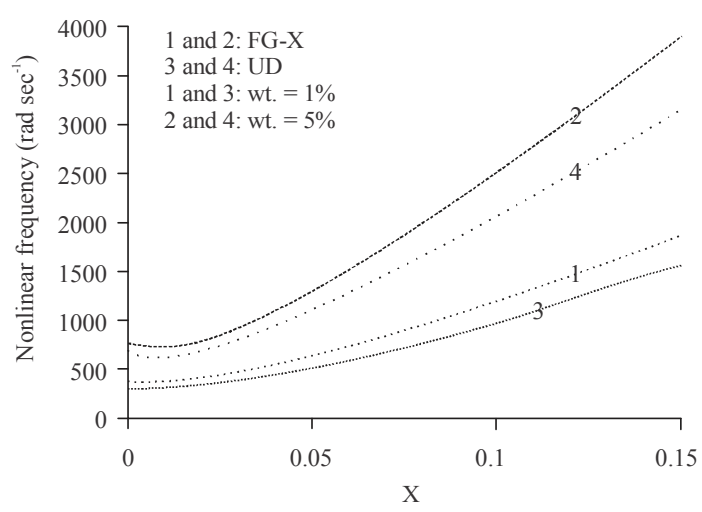

Fig. 4: Effect of weight fraction (wt.) and distribution patterns on nonlinear frequency-amplitude relation

patterns. As predicted, the nonlinear vibration frequencies increase with the increasing in the weight fraction. Also, the nonlinear frequencies for (FG_X) distribution pattern are greater than for (UD) uniform distribution style. Figure 5 shows the effect of stiffeners thickness on the nonlinear frequencies with (FG_X) distribution pattern. It is obviously that the small increasing in the stiffener thickness lead to considerable increasing in the nonlinear frequencies. Figure 6 and 7 demonstrates the effect of spacing (s1) and height (h1) of stiffeners on the nonlinear frequency-amplitude relation, respectively. It's clearly that the effezct of parameter (s1) on the nonlinear frequencies is greater than the influence of parameter (h1) Table 1.

Results of nonlinear dynamic behaviour: In this part, the effects of the GPLs distribution pattern, weight fraction of GPLs, exciting force, imperfection and geometric parameters of stiffeners on the nonlinear dynamic response of the FG-GPLs Shell panel will be 
J. Eng. Applied Sci., 14 (Special Issue 8): 10349-10359, 2019

Table 1: The natural frequencies of $(\mathrm{rad} / \mathrm{s})$ of FG-GPLs cylindrical panels

\begin{tabular}{|c|c|c|c|c|}
\hline$\underline{\mathrm{R}(\mathrm{m})}$ & GPLs distribution pattern & wt.\% & Stiffened $(m, n)$ & Unstiffened $(\mathrm{m}, \mathrm{n}$ \\
\hline & & 1 & $1079.4(1,2)$ & $460.648(1,3)$ \\
\hline & FG_X & 3 & $1746.7(1,2)$ & $745.731(1,3)$ \\
\hline & & 5 & $2223.0(1,2)$ & $948.924(1,3)$ \\
\hline & & 1 & $980.1095(1,2)$ & $416.725(1,3)$ \\
\hline & UD & 3 & $1561.9(1,2)$ & $664.0535(1,3)$ \\
\hline & & 5 & $1980.6(1,2)$ & $842.0113(1,3)$ \\
\hline \multirow[t]{8}{*}{1.5} & & 1 & $849.29(1,2)$ & $367.6279(1,3)$ \\
\hline & FG_O & 3 & $1299.9(1,2)$ & $570.5389(1,3)$ \\
\hline & & 5 & $1629.8(1,2)$ & $718.7269(1,3)$ \\
\hline & & 1 & $1030.3(1,2)$ & $298.603(1,2)$ \\
\hline & FG_X & 3 & $1670.8(1,2)$ & $480.535(1,2)$ \\
\hline & & 5 & $2127.4(1,2)$ & $610.855(1,2)$ \\
\hline & & 1 & $919.65(1,2)$ & $281.995(1,2)$ \\
\hline & UD & 3 & $1465.5(1,2)$ & $449.554(1,2)$ \\
\hline \multirow[t]{11}{*}{3.0} & & 5 & $1858.3(1,2)$ & $570.2707(1,2)$ \\
\hline & & 1 & $766.1391(1,2)$ & $264.39(1,2)$ \\
\hline & FG_O & 3 & $1155.9(1,2)$ & $416.249(1,2)$ \\
\hline & & 5 & $1443.0(1,2)$ & $526.471(1,2)$ \\
\hline & & 1 & $430.469(1,1)$ & $241.549(1,2)$ \\
\hline & FG_X & 3 & $693.664(1,1)$ & $390.519(1,2)$ \\
\hline & & 5 & $882.0346(1,1)$ & $496.815(1,2)$ \\
\hline & & 1 & $403.202(1,1)$ & $220.704(1,2)$ \\
\hline & UD & 3 & $642.6539(1,1)$ & $351.730(1,2)$ \\
\hline & & 5 & $815.0629(1,1)$ & $446.0347(1,2)$ \\
\hline & & 1 & $372.294(1,1)$ & $197.697(1,2)$ \\
\hline \multirow[t]{2}{*}{5.0} & FG_O & 3 & $583.0575(1,1)$ & $307.98(1,2)$ \\
\hline & & 5 & $736.24(1,1)$ & $388.398(1,2)$ \\
\hline
\end{tabular}

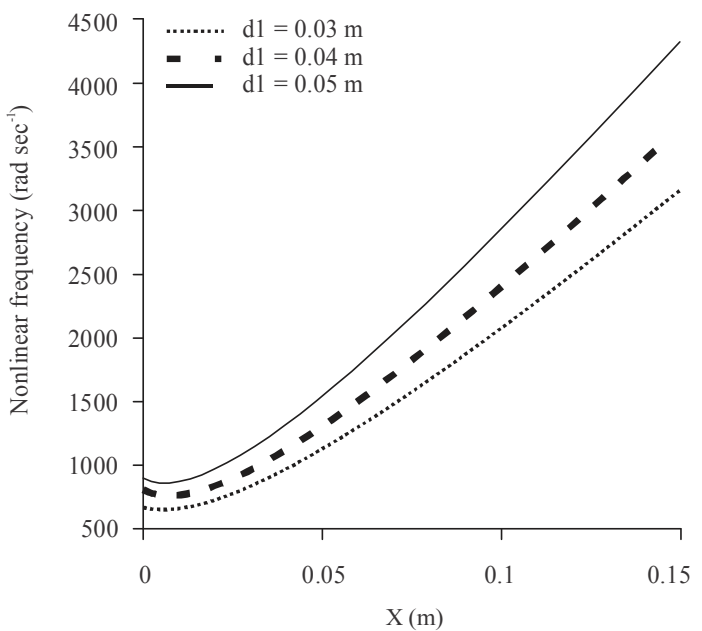

Fig. 5: Effect of stiffeners thickness on frequencyamplitude relation

consider. Figure 7 illustrates the influence of weight fraction of graphene on the nonlinear dynamic response of FG-GPLs Shellpanel.

It can observe that the amplitude of nonlinear vibration of the panel increases with the increasing in the weight fraction. The effect of distribution pattern for GPLs on the vibration amplitude of FG-GPLs Shellis demonstrates in Fig. 8. It's evident that the vibration amplitude for (FG_X) distribution pattern is the lower and (FG_O) is the higher while the amplitude for the Uniform Distribution pattern (UD) lies in between. Figure 9

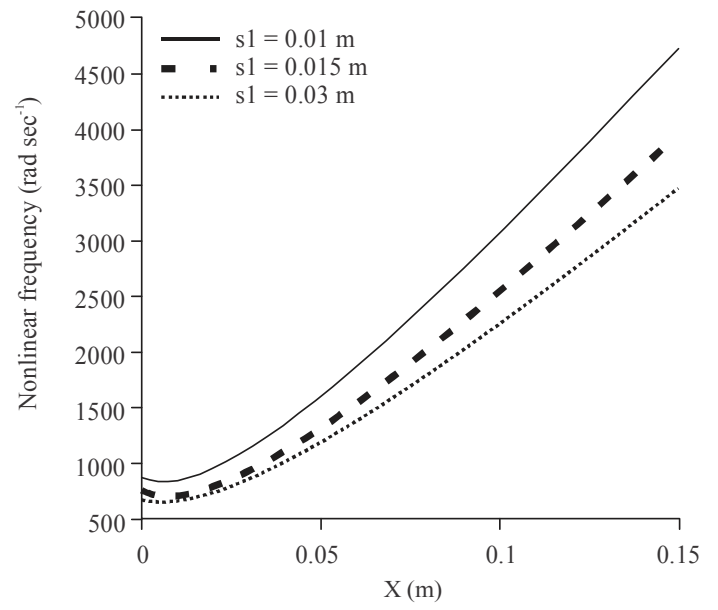

Fig. 6: Effect of stiffeners spacing on frequencyamplitude relation

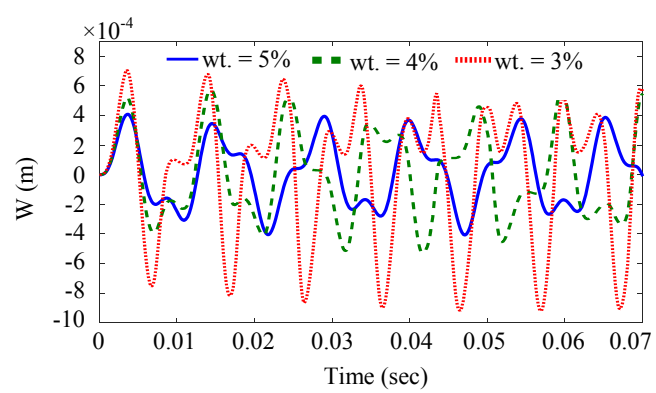

Fig. 7: Effect of weight fraction (wt.) on the dynamic response of FG-GPLs cylindrical panel 


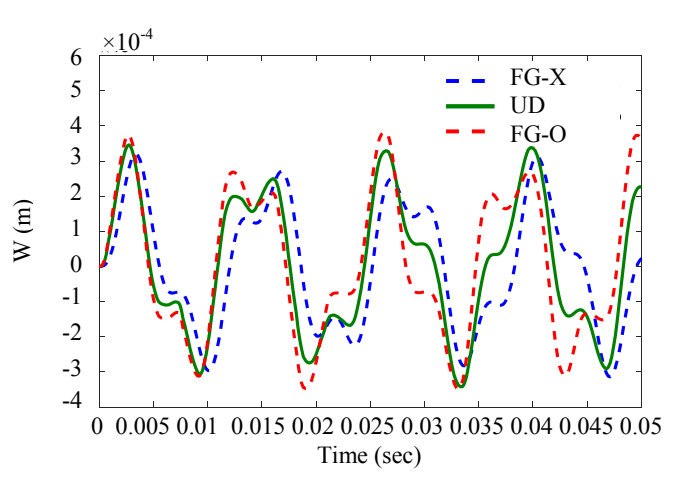

Fig. 8: Effect of distribution pattern on the dynamic response of FG-GPLs cylindrical panel

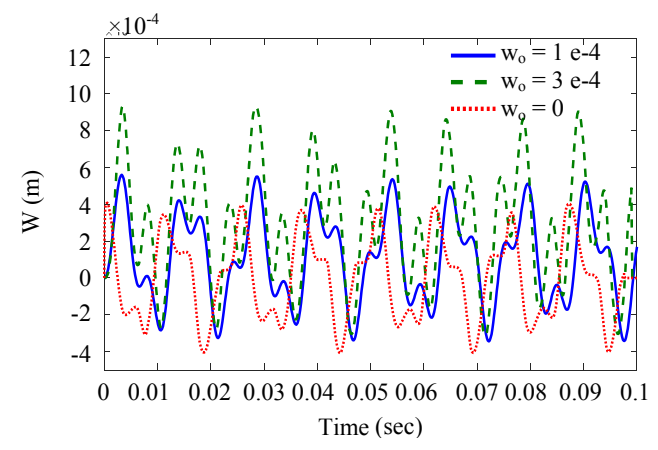

Fig. 9: Effect of imperfection parameter on the dynamic response of FG-GPLs cylindrical panel

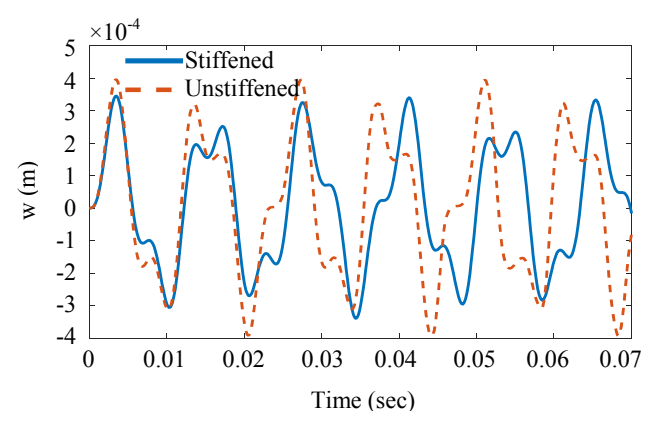

Fig. 10: Effect of stiffeners on the dynamic response of FG-GPLs cylindrical panel

characterizes the nonlinear dynamic response of eccentrically stiffened FG-GPLs Shellpanel under the effect of the imperfection parameter. It's clear that the vibration amplitude is greatly affected with the imperfection param eter. Figure 10 displays the influences of stiffeners on the vibration response for the Shellpanels reinforced with FG graphene sheets.

The outcome displays that the stiffeners robustly decrease the vibration response of the Shell panel. Figure 11 points to the influence of the exciting force on

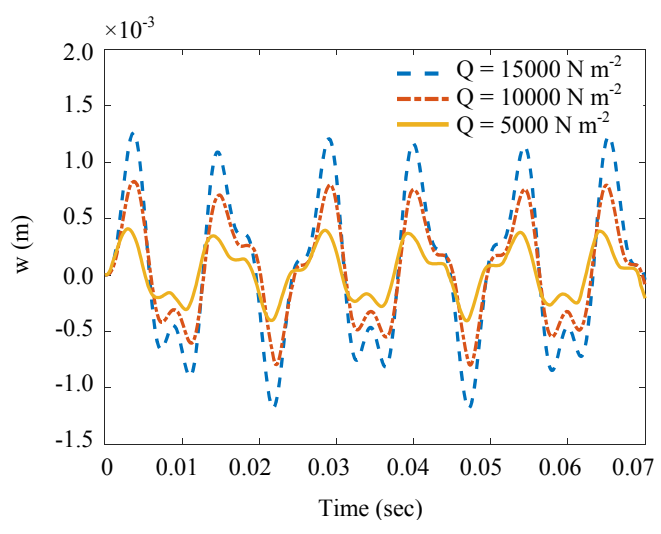

Fig. 11: Effect of exciting force on the dynamic response of FG-GPLs cylindrical panel

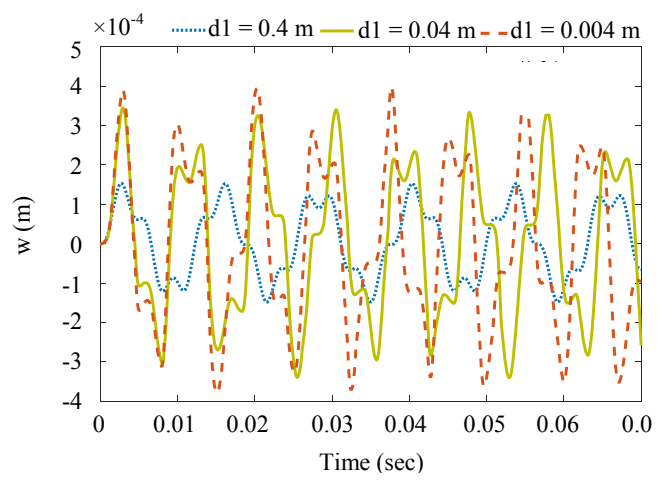

Fig. 12: Effect of stiffeners thickness on the dynamic response of FG-GPLs cylindrical panel

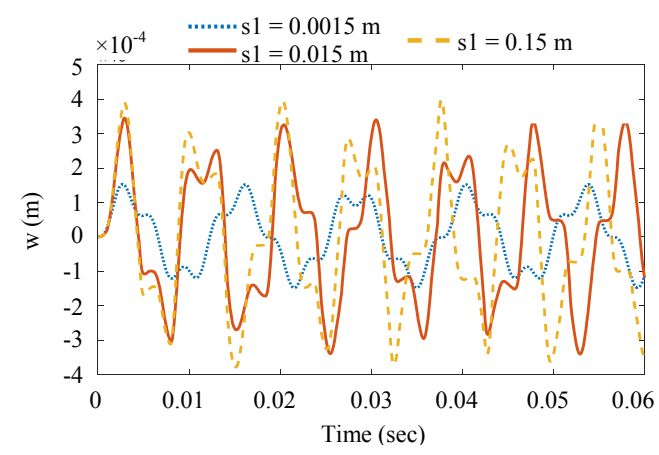

Fig. 13: Effect of stiffeners spacing on the dynamic response of FG-GPLs cylindrical panel

the vibration amplitude for the Shellpanels. It can be seen that the FG-GPLs Shellpanel variation amplitudes increase when the exciting force increase. Figure 12-14 indicate the effect of geometric parameters of stiffeners on the dynamic response of FG-GPLs Shell panels. From Fig. 13 the vibration amplitude decreases when increasing stiffener thickness (d1). Figure 14 characterizes the effect 


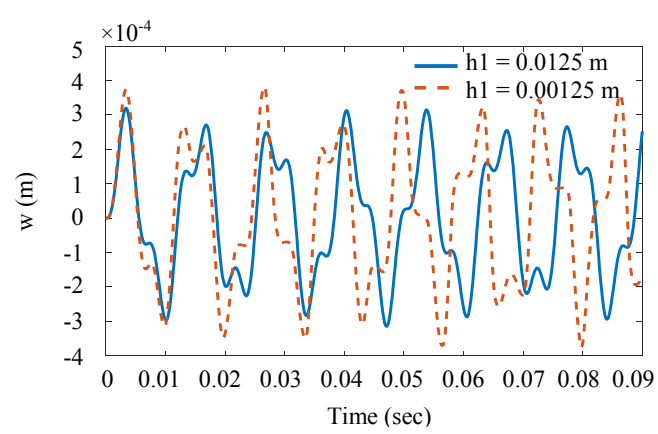

Fig. 14: Effect of stiffeners height on the dynamic response

of stiffener spacing on the nonlinear dynamic behaviour for the FG-GPLs Shellpanels and the panel fluctuates weakly when the factor (s1) is decreased. Finally, Fig. 14 shows the influence of stiffener height the vibration response of the shell. It's evident that the increasing the parameter (h1) leads to decreasing the vibration amplitude.

\section{CONCLUSION}

A classical Shelltheory coupled with Von Karman nonlinear terms has been employed in the formulization of the governing equations of $\mathrm{FG}$ graphene-reinforced composite eccentrically stiffened cylindrical Shellpanels. Galerkin solution based technique is utilized to determine the fundamental frequencies, nonlinear frequencies as well as the dynamic response for simply supported cylindrical panels under the effect of dynamic loading. The impact of graphene distribution style, weight fraction, stiffeners and geometric parameters of stiffeners, imperfection factor and the exciting force on the nonlinear dynamic behaviour of the Shellpanel has been investigated. It is that reinforcing the stiffeners with graphene sheets plays an important role in increasing the nonlinear frequencies and decreasing the dynamic deflections an addendum a very small amount of graphene sheets able to considerable increasing nonlinear frequencies and decreasing the vibration amplitude graphene distribution pattern (i.e., FG_X) is the effective way to enhance the Shellpanels.

\section{REFERENCES}

Bich, D.H. and V.D. Long, 2010. Non-linear dynamical analysis of imperfect functionally graded material shallow shells. Vietnam J. Mech., 32: 1-14.

Bich, D.H., D. van Dung and V.H. Nam, 2012. Nonlinear dynamical analysis of eccentrically stiffened functionally graded cylindrical panels. Compos. Struct., 94: 2465-2473.
Bich, D.H., D. van Dung, V.H. Nam and N.T. Phuong, 2013. Nonlinear static and dynamic buckling analysis of imperfect eccentrically stiffened functionally graded circular cylindrical thin shells under axial compression. Int. J. Mech. Sci., 74: 190-200.

Chandra, Y., R. Chowdhury, F. Scarpa, S. Adhikari and J. Sienz et al., 2012. Vibration frequency of graphene based composites: A multiscale approach. Mater. Sci. Eng. B., 177: 303-310.

Debelak, B. and K. Lafdi, 2007. Use of exfoliated graphite filler to enhance polymer physical properties. Carbon, 45: 1727-1734.

Duc, N.D. and P.T. Thang, 2014. Nonlinear response of imperfect eccentrically stiffened ceramic-metal-ceramic FGM thin circular cylindrical shells surrounded on elastic foundations and subjected to Axial compression. Compos. Struct., 110: 200-206

Duc, N.D. and T.Q. Quan, 2013. Nonlinear postbuckling of imperfect eccentrically stiffened P-FGM double curved thin shallow shells on elastic foundations in thermal environments. Compo. Struct., 106: 590-600.

Duc, N.D., P.H. Cong and V.D. Quang, 2016. Nonlinear dynamic and vibration analysis of piezoelectric eccentrically stiffened FGM plates in thermal environment. Int. J. Mech. Sci., 115: 711-722.

Dung, D.V. and V.H. Nam, 2014. Nonlinear dynamic analysis of eccentrically stiffened functionally graded circular cylindrical thin shells under external pressure and surrounded by an elastic medium. Eur. J. Mech. A. Solids, 46: 42-53.

Fang, M., K. Wang, H. Lu, Y. Yang and S. Nutt, 2009. Covalent polymer functionalization of graphene nanosheets and mechanical properties of composites. J. Mater. Chem., 19: 7098-7105.

Geng, Y., M.Y. Liu, J. Li, X.M. Shi and J.K. Kim, 2008. Effects of surfactant treatment on mechanical and electrical properties of CNT/epoxy nanocomposites. Compos. Part A. Applied Sci. Manuf., 39: 1876-1883.

Li, Q., J.W. Kim, T.H. Shim, Y.K. Jang and J.H. Lee, 2008. Positive temperature coefficient behavior of the graphite nanofibre and carbon black filled high-density polyethylene hybrid composites. Adv. Mater. Res., 47: 226-229.

Liao, S.H., C.Y. Yen, C.C. Weng, Y.F. Lin and C.C.M. Ma et al., 2008. Preparation and properties of carbon nanotube/polypropylene nanocomposite bipolar plates for polymer electrolyte membrane fuel cells. J. Power Sources, 185: 1225-1232.

Milani, M.A., D. Gonzalez, R. Quijada, N.R. Basso, M.L. Cerrada, D.S. Azambuja and G.B. Galland, 2013. Polypropylene/graphene nanosheet nanocomposites by in situ polymerization: Synthesis, characterization and fundamental properties. Compos. Sci. Technol., 84: 1-7. 
Montazeri, A. and H. Rafii-Tabar, 2011. Multiscale modeling of grapheme and nanotube-based reinforced polymer nanocomposites. Phys. Lett. A., 375: 4034-4040.

Najafizadeh, M.M., A. Hasani and P. Khazaeinejad, 2009. Mechanical stability of functionally graded stiffened cylindrical shells. Applied Math. Modell., 33: 1151-1157.

Novoselov, K.S., A.K. Geim, S. V. Morozov, D. Jiang and $\mathrm{Y}$. Zhang et al., 2004. Electric field effect in atomically thin carbon films. Science, 306: 666-669.

Putz, K.W., O.C. Compton, M.J. Palmeri, S.T. Nguyen and L.C. Brinson, 2010. High nanofiller content graphene oxide polymer nanocomposites via vacuumassisted selfassembly. Adv. Funct. Mater., 20: 3322-3329.

Rafiee, M.A., J. Rafiee, Z.Z. Yu and N. Koratkar, 2009. Buckling resistant graphene nanocomposites. Applied Phys. Lett., Vol. 95, No. 22. 10.1063/1.3269637

Reddy, J.N. and C.F. Liu, 1985. A higher-order shear deformation theory of laminated elastic shells. Int. J. Eng. Sci., 23: 319-330.

Rissanou, A., A. Power and V. Harmandaris, 2015. Structural and dynamical properties of polyethylene/graphene nanocomposites through molecular dynamics simulations. Polymers, 7: 390-417.

Shen, H.S., 2001. Thermal postbuckling behavior of imperfect shear deformable laminated plates with temperature-dependent properties. Comput. Methods Applied Mech. Eng., 190: 5377-5390.
Shen, H.S., Y. Xiang and F. Lin, 2017. Nonlinear vibration of functionally graded graphene-reinforced composite laminated plates in thermal environments. Comput. Methods Applied Mech. Eng., 319 : 175-193.

Shen, H.S., Y. Xiang and F. Lin, 2017. Thermal buckling and postbuckling of functionally graded graphene-reinforced composite laminated plates resting on elastic foundations. Thin-Walled Struct., 118: 229-237.

Song, M., J. Yang, S. Kitipornchai and W. Zhu, 2017. Buckling and postbuckling of biaxially compressed functionally graded multilayer graphene nanoplatelet-reinforced polymer composite plates. Int. J. Mech. Sci., 131: 345-355.

Song, M., S. Kitipornchai and J. Yang, 2017. Free and forced vibrations of functionally graded polymer composite plates reinforced with graphene nanoplatelets. Compos. Struct., 159: 579-588.

Spitalsky, Z., D. Tasis, K. Papagelis and C. Galiotis, 2010. Carbon nanotube-polymer composites: Chemistry, processing, mechanical and electrical properties. Prog. Polymer Sci., 35: 357-401.

Wang, H., H. Zhang, W. Zhao, W. Zhang and G. Chen, 2008. Preparation of polymer/oriented graphite nanosheet composite by electric field-inducement. J. Compos. Sci. Technol., 68: 238-243.

Wang, Y., J. Yu, W. Dai, Y. Song, D. Wang, L. Zeng and N. Jiang, 2015. Enhanced thermal and electrical properties of epoxy composites reinforced with graphene nanoplatelets. Polym. Compos., 36: $556-565$.

Yang, J., H. Wu and S. Kitipornchai, 2017. Buckling and postbuckling of functionally graded multilayer graphene platelet-reinforced composite beams. Compos. Struct., 161: 111-118. 